\title{
Health Facility Capacity to Provide Maternal and Newborn Healthcare Services in Unguja
}

\author{
Rukia Rajab Bakar ${ }^{1,2,3}$, Rachel N Manongi ${ }^{1,2}$ \& Blandina T. Mmbaga ${ }^{1,4,5}$ \\ ${ }^{1}$ Kilimanjaro Christian Medical University College, Tumaini University, Moshi, Tanzania \\ ${ }^{2}$ Institute of Public Health, Kilimanjaro Christian Medical University College, Moshi, Tanzania \\ ${ }^{3}$ Department of Nursing and Midwifery, School of Health and Medical Sciences, The State University of Zanzibar \\ (SUZA), Zanzibar, Tanzania \\ ${ }^{4}$ Kilimanjaro Clinical Research Institute (KCRI), Moshi, Tanzania \\ ${ }^{5}$ Kilimanjaro Christian Medical Centre (KCMC), Moshi, Tanzania \\ Correspondence: Rukia Rajab Bakar, Department of Nursing and Midwifery, School of Health and Medical \\ Sciences, The State University of Zanzibar (SUZA), Zanzibar, Tanzania. Tel: 255-77-745-7234. E-mail: \\ rrbakar2012@yahoo.com
}

Received: April 16, 2019 Accepted: June 24, 2019 Online Published: August 20, 2019

doi:10.5539/gjhs.v11n10p120 URL: https://doi.org/10.5539/gjhs.v11n10p120

\begin{abstract}
Globally, every year 529,000 maternal deaths occur, $99 \%$ of which in developing countries with majority being in Sub-Saharan Africa. Maternal, Newborn and Child Health $(\mathrm{MNCH})$ services depend on the accessibility, availability and quality of antenatal care (ANC), delivery and postnatal services. The aim of this study was to assess the health facilities' capacity and readiness to provide $\mathrm{MNCH}$ services in Unguja Island, Zanzibar.

A facility-based cross-sectional survey was conducted from May to June 2015 at public health facilities providing MNCH services. Data was collected by using the modified Service Availability and Readiness Assessment tool.

Eighteen health facilities were assessed, two-thirds $(66.7 \%, \mathrm{n}=12)$ of which were offering both maternity and reproductive and child health $(\mathrm{RCH})$ services, 4 (22.2\%) $\mathrm{RCH}$ services only, and 2 (11.1\%) maternity services only. Readiness score for ANC services was $66 \%$ with high readiness scores in diagnostics services $(89 \%)$ and equipment (69\%). Overall, 14\% offered all seven signal functions. Overall, delivery service readiness score was $48 \%$. Overall readiness for comprehensive emergency obstetric and neonatal care services was $13 \%$. Staff training and guidelines readiness score was $11 \%$, while medicine and commodities score was $9 \%$.

The health facilities' readiness in providing $\mathrm{MNCH}$ services remains inadequate in Unguja Island. Readiness in providing services was low for delivery and emergency obstetric and neonatal care services. Basic and advanced delivery services need to be improved in parallel with provision of necessary equipment, medicines and commodities and staff training for better $\mathrm{MNCH}$ service delivery.
\end{abstract}

Keywords: emergency obstetric care, health facilities, maternal and newborn healthcare services, Unguja Island, Zanzibar

\section{Introduction}

In Africa maternal healthcare service delivery is a huge challenge. This is compounded by inadequate and poor distribution healthcare facilities, lack of equipment and drugs (Obi et al., 2013; Singh et al., 2014). Zanzibar, like other African countries, also faces some challenges in addressing maternal and newborn healthcare services (WHO, 2014). The performance of the health sector remains unsatisfactory even though geographical coverage of the health facilities is considered equitably distributed to all regions and districts, and easily accessible to $95 \%$ of the population. Accessibility to health services is constrained mainly by poor quality of services due to lack of equipment and qualified staff (MoHZ, 2009). High maternal mortality still remains a serious challenge in Zanzibar with estimated Maternal Mortality Ratio (MMR) of 307 deaths per 100,000 live births (Herklots et al., 2017; HMIS, 2016; NBS, 2012). The major challenges in the reduction of maternal mortality include limited access to quality health services, a weak referral system and poor health-seeking behaviour among women (WHO, 2014).

In order to reduce preventable maternal mortality, a global action has been initiated through implementation of 
Sustainable Development Goals (SDGs), which target for reducing global MMR to less than 70 deaths per 100,000 live births by 2030 (WHO, 2015). However, achievement of SDG targets is ambitious for most high-mortality countries (Alkema et al., 2016). In previous series of 4 years, Zanzibar did not meet the Millennium Development Goals (MDG) target which was 170 per 100,000 live births by 2015, but it might reach the SDG target by 2030, if extra effort and commitment is taken by various stakeholders (HMIS, 2016).

Most maternal and newborn deaths occur on the day of birth, therefore essential lifesaving interventions need to be delivered at facilities with capacity to provide support of normal labor and birth as well as basic or comprehensive emergency obstetric and newborn care (Campbell \& Graham, 2006). It is therefore crucially important to increase women's access to quality care before, during and after childbirth (WHO, 2017). Availability and utilisation of quality emergency obstetric and neonatal care $(E m O N C)$ services is one of the essential interventions for reduction of a substantial proportion of maternal and newborn mortality (Bakari et al., 2015; Chi et al., 2015; Paxton et al., 2005). These are services necessary to save life and are most useful when complications occur during pregnancy, childbirth and after birth (Chi et al., 2015). According to the United Nations (UN) recommendations, there should be at least one comprehensive and four basic EmONC facilities per 500,000 population (WHO, UNFPA, \& UNICEF, 2009).

If services are available and in adequate supply, then the opportunity to obtain healthcare exists, and a population may have 'access' to services (MoHSW, 2010). Adequate availability of infrastructure, functional transport and communication systems for timely and quick referral of emergency patients to the next higher level of healthcare facility and availability of essential drugs, equipment, and supplies play a major role in delivering high-quality EmONC and other MNCH-related services.

The improvement of maternal and newborn health is a key priority for the Government of Zanzibar. Many efforts have been made in Zanzibar to reduce maternal and neonatal deaths. As in many other countries, Zanzibar has made great strides in implementing EmONC in the last few years. The efforts have focused on increasing the proportion of births attended by skilled health providers and the coverage of facility-based maternity services and emergency obstetric care. Currently about $50 \%$ of deliveries in Zanzibar occur in health facilities (Fakih et al., 2016). Despite the efforts made, the current situation of maternal and newborn care services does not meet the obstetric needs as set by the UN process indicators. Although problems of providing very basic equipment and a minimum number of staff continue, there are sufficient numbers of CEmONC facilities, but the distribution in Unguja is not adequate and some healthcare facilities providing delivery services do not meet BEmONC criteria (MoHZ, 2008).

This study assessed the health facilities on service availability and readiness of maternal and newborn healthcare services. Service availability refers to the physical presence of essential maternal and newborn healthcare services, while service readiness refers to the presence of functioning equipment, supplies, medicine that are in-stock and non-expired, trained staff, and current guidelines to provide maternal and newborn healthcare services. Both are a prerequisite to providing good-quality healthcare services.

\section{Methods}

\subsection{Study Design}

This was a facility-based cross-sectional survey, which was conducted between May and June 2015 in Unguja Island, Zanzibar.

\subsection{Study Area}

The study was conducted at Unguja Island, Zanzibar. Unguja Island is one of the two Islands in Zanzibar; the other being Pemba Island. Unguja Island is located 40 kilometres East of Tanzania Mainland. Unguja Island has a total of six districts with a population of 896,721 which is mostly concentrated in the Urban-West districts (NBS, 2013).

\subsubsection{Selection of Study Sites and Health Facilities}

A simple random sampling method was used to select four districts out of six. The selected districts were North A, North B, West and Urban districts. These districts have high maternal mortality compared to other districts in Unguja Island (NBS, 2012). These districts have the total of 57 public health facilities, among which eighteen facilities were selected randomly to represent other facilities in the study area. We selected only public facilities because majority of people in the general community utilise them and also the healthcare services of the public facilities are the mirror which reflects the general health of the people in the country as well. The study involved facilities that provide maternal and newborn healthcare services. 


\subsubsection{Healthcare System in Zanzibar}

The Zanzibar healthcare system is made up of public and private sectors, and the healthcare delivery services are categorized into three levels, namely 1) Primary level: This is the lowest level of healthcare delivery in the public healthcare structure. It comprises the Primary Health Care Unit (PHCU), Primary Health Care Unit plus (PHCU+) and Primary Health Care Centres (PHCC). PHCU+ provides additional services such as delivery, dental, laboratory and pharmacy services, which are not in PHCU. 2) Secondary level: Is the referral point from primary health facilities comprising of district hospitals. All these are located in Pemba and none in Unguja Island. 3) Tertiary level: Mnazi Mmoja Hospital is the only tertiary hospital which is located in Unguja town and it provides the referral services for Zanzibar, including two specialised hospitals known as Mwembeladu maternity home and Kidongochekundu mental hospital. The population of Zanzibar lives within less than five kilometers from public health facilities, which confirms to WHO standard (5-10 km) (MoHZ, 2016). In Zanzibar, according to the health system guidelines, all hospitals, Primary Health Care Centres (PHCC) and Primary Health Care Unit plus (PHCU+) are required to conduct delivery services, including BEmONC services. In addition, all hospitals and PHCCs are required to provide CEmONC services.

\subsection{Data Collection Tool}

The World Health Organization (WHO) Service Availability and Readiness Assessment (SARA) tool was used to collect data during assessment of the health facilities in order to address critical data gaps in service availability and readiness for the facilities to provide $\mathrm{MNCH}$ care services. The tool is a comprehensive approach to evaluate different areas of health care services including maternal and newborn health care services, and is widely used globally. Also, it is a reliable standard tool to monitor health facilities and services delivery. In this study, the WHO's SARA tool was adopted and modified to focus mainly on service availability and service readiness specific for maternal and newborn healthcare services. The tool was divided into four main sections; 1) Health facility information 2) Antenatal care services, 3) Basic delivery services, and 4) Emergency obstetric and newborn care services. The tool was used to collect data of two main domains, namely: 1.) Availability (physical presence of the delivery of services) and 2.) Readiness (capacity to offer a specific service). The capacity to provide that service was measured through consideration of availability of tracer items that include trained staff, guidelines, equipment, diagnostic capacity, and medicines and commodities.

\subsection{Data Collection Methods}

The health facilities were assessed through observation method based on service availability and functionality of equipment and availability of supplies, medicines and commodities in order to assess facility availability and readiness of $\mathrm{MNCH}$ care services. Interviews were done to the facilities in-charges in order to supplement some of the required information of the surveyed facilities. Two nurses with research experience in monitoring and evaluation of $\mathrm{MNCH}$ services were recruited and trained on the use of the tool and conducting the health facility survey. The collected information was evaluated based on facility level, overall capacity to provide MNCH services with regard to standard guidelines.

\subsection{Data Analysis}

Data was checked for completeness before being coded. The coded data were entered in the computer to create electronic database and then, analysed using Statistical Package for Social Sciences (SPSS) software, version 22.0 and Microsoft Excel software was used for tabulation and graphing of results. The components of service availability were analysed based on physical availability of services. Service readiness analysis described the availability of essential inputs needed, called tracer items, to deliver service-specific interventions across five areas: (i) trained staff and (ii) guidelines; (iii) functioning equipment; (iv) diagnostic capacities and (v) essential medicines and commodities. During analysis, descriptive statistics were computed. Within each area, a mean score was calculated and tracer items were given equal weight. Finally, data were summarized and the results were displayed using frequency distributions and charts.

\subsection{Ethical Considerations}

The study was approved by the Kilimanjaro Christian Medical University College Research and Ethical Review Committee (CRERC), (certificate number 677) and Zanzibar Medical Research Council (ZAMREC). Permission to conduct research was obtained from the respective District Medical Officers and in-charges of each health facility surveyed. 


\section{Results}

\subsection{Health Facilities Information}

Eighteen health facilities were included in the study, of which $11(61 \%)$ were primary health centre units plus (PHCU+), 4 (22.2\%) primary health centre units (PHCUs), 1 (5.6\%) tertiary hospital, 1 (5.6\%) special maternity hospital and 1 (5.6\%) primary health care centre (PHCC). All facilities were managed by the government. Nine $(50 \%)$ were providing antenatal care (ANC) services, including other reproductive and child health (RCH) services, 7 (39\%) were offering both ANC and delivery services while $2(11 \%)$ were offering only delivery services.

\subsection{Distribution of Healthcare Personnel According to Level of Health Facility}

Doctors were mostly found in the tertiary hospital, PHCC and the special maternity hospital. Registered nurse midwives were more distributed in PHCUs than in other levels of health facilities. The few $\mathrm{MCH}$ aides were mainly found in primary health care units (PHCUs). Clinical officers were mainly clustered in PHCC and PHCUs (Table 1).

Table 1. Distribution of healthcare personnel according to level of health facility

\begin{tabular}{llllll}
\hline Type of health facility & Doctors & Clinical officers & Nurses Midwives & PHN B & MCH Aides \\
\hline All & 6 & 28 & 72 & 51 & 4 \\
Tertiary hospital & 3 & 0 & 18 & 4 & 0 \\
Special maternity hospital & 1 & 0 & 12 & 3 & 0 \\
Primary healthcare centre & 2 & 9 & 9 & 0 & 0 \\
Primary healthcare units plus & 0 & 16 & 20 & 35 & 2 \\
Primary healthcare units & 0 & 3 & 13 & 9 & 2 \\
\hline
\end{tabular}

\subsection{Antenatal Care Service Availability and Readiness}

The availability of antenatal care services of all surveyed facilities during study was 16 (89\%). Readiness to provide ANC services were assessed based on availability of 10 tracer items, which categorized into staff training and guidelines, equipment, medicines and commodities and diagnostics services. Overall readiness score in provision of antenatal care services was (66\%), with the highest readiness scores observed in diagnostics (89\%) and equipment (69\%), while the lower service readiness scores were observed for the trained staff and guidelines $(56 \%)$ and medicines and commodities (47\%) (Figure 1).

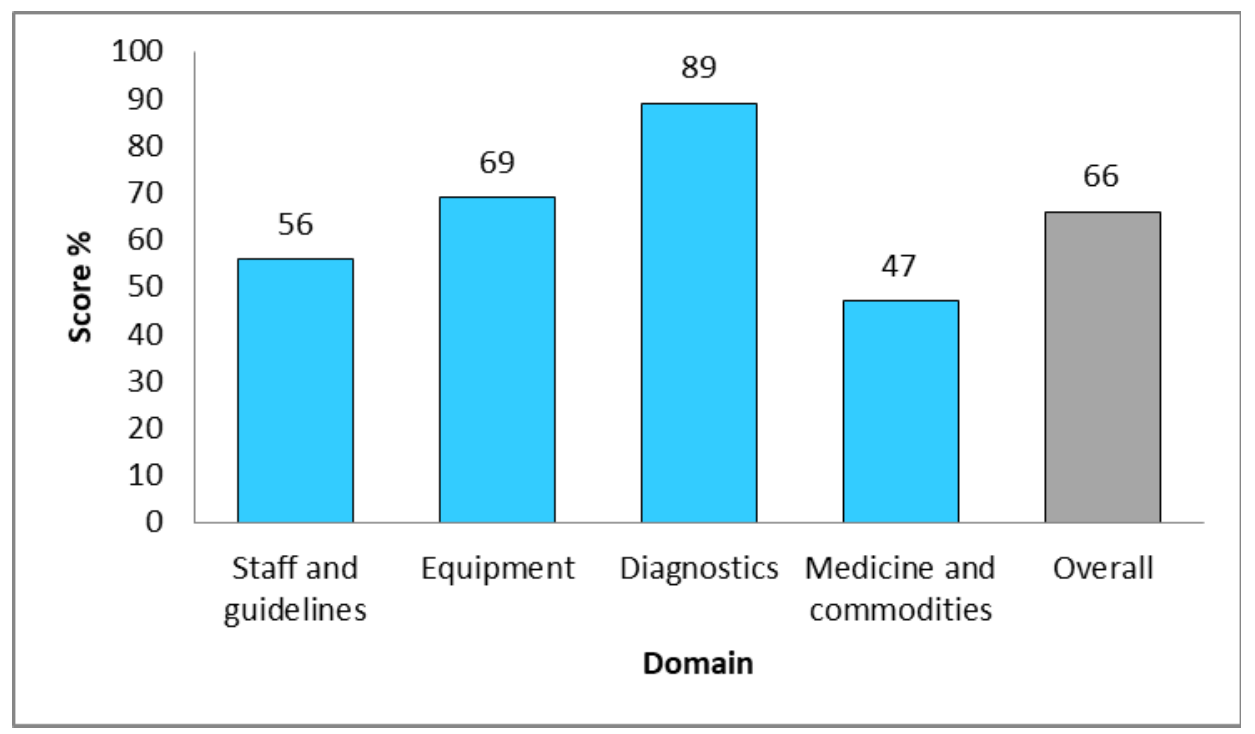

Figure 1. Readiness scores of antenatal care domains 
Relatively higher scores for all categories of service readiness were demonstrated in PHCC. The lowest readiness scores for equipment (59\%) and medicines and commodities (48\%) were demonstrated in PHCU+, while for staff training and guidelines, medicines and commodities the lowest readiness scores were observed in PHCUs (50\%) and $(42 \%)$, respectively (Figure 2 ).

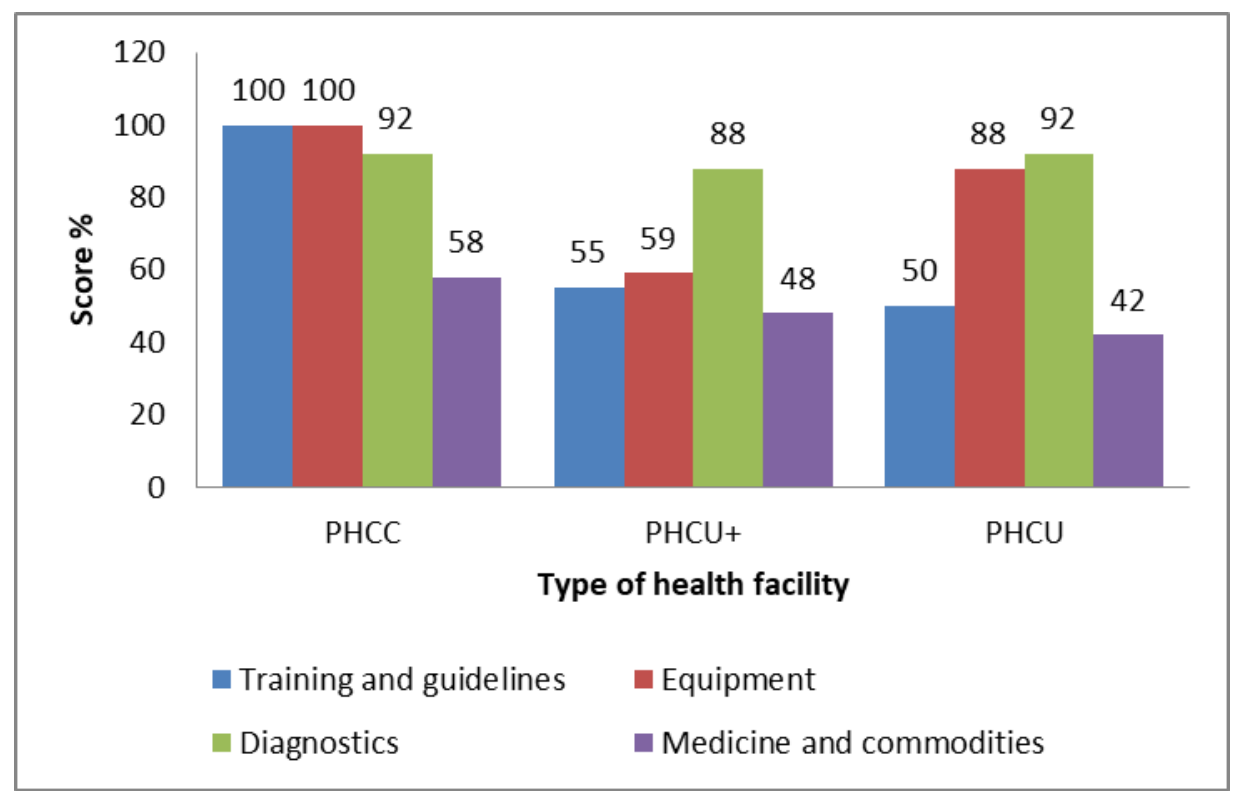

Figure 2. Antenatal care domain scores according to level of health facility

\subsection{Availability and Readiness of Basic Delivery Services}

The services readiness for the facilities to provide basic delivery services was assessed on 16 tracer items (Table 2). These tracer items were categorized into equipment, and medicines and commodities. Of the 18 surveyed facilities, $9(50 \%)$ had the basic delivery services available, of which $33 \%$ of the services were available in urban settings.

The overall service readiness was $66 \%$, with high score in medicines and commodities (81\%) and the lowest score was in equipment (52\%), (Figure 3).

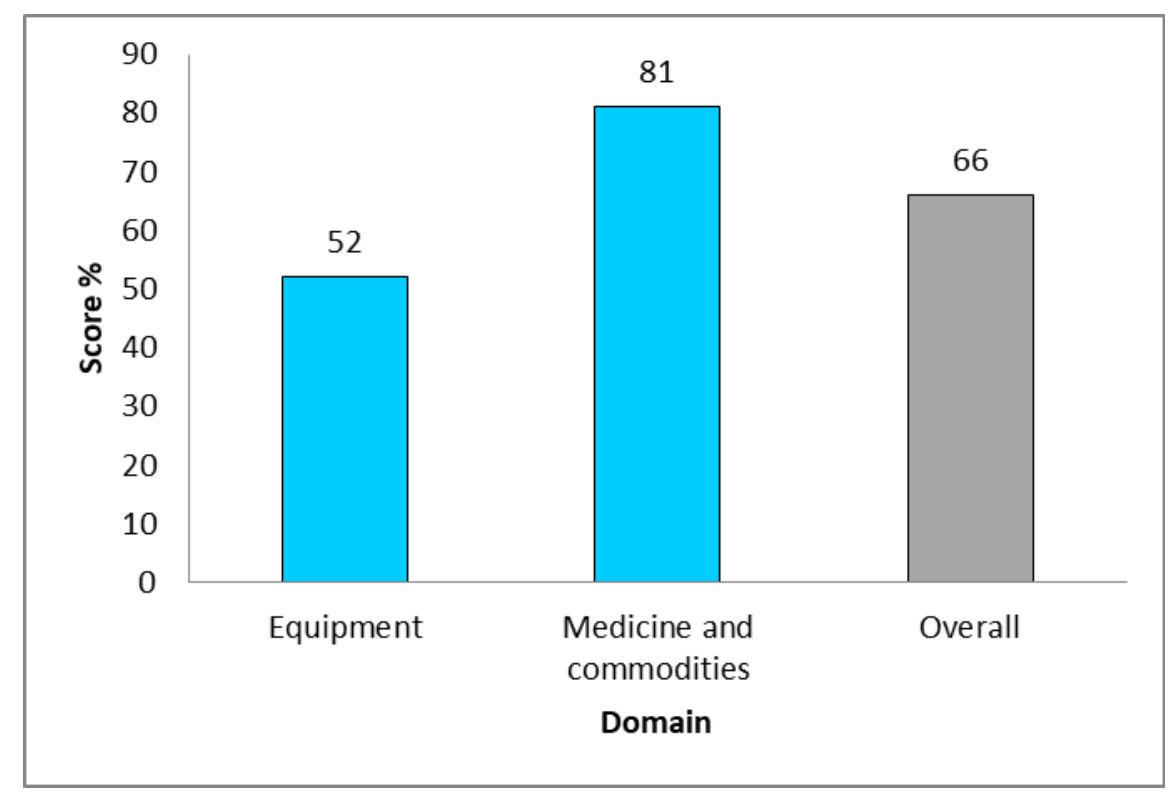

Figure 3. Readiness score for provision of basic delivery services 
The service readiness score for equipment was higher $(78 \%)$ in PHCC and lower in the special maternity hospitalandPHCUs+ (44\% and $46 \%$ respectively) while service readiness score for medicines and commodities were almost similar across all facilities (Figure 4).

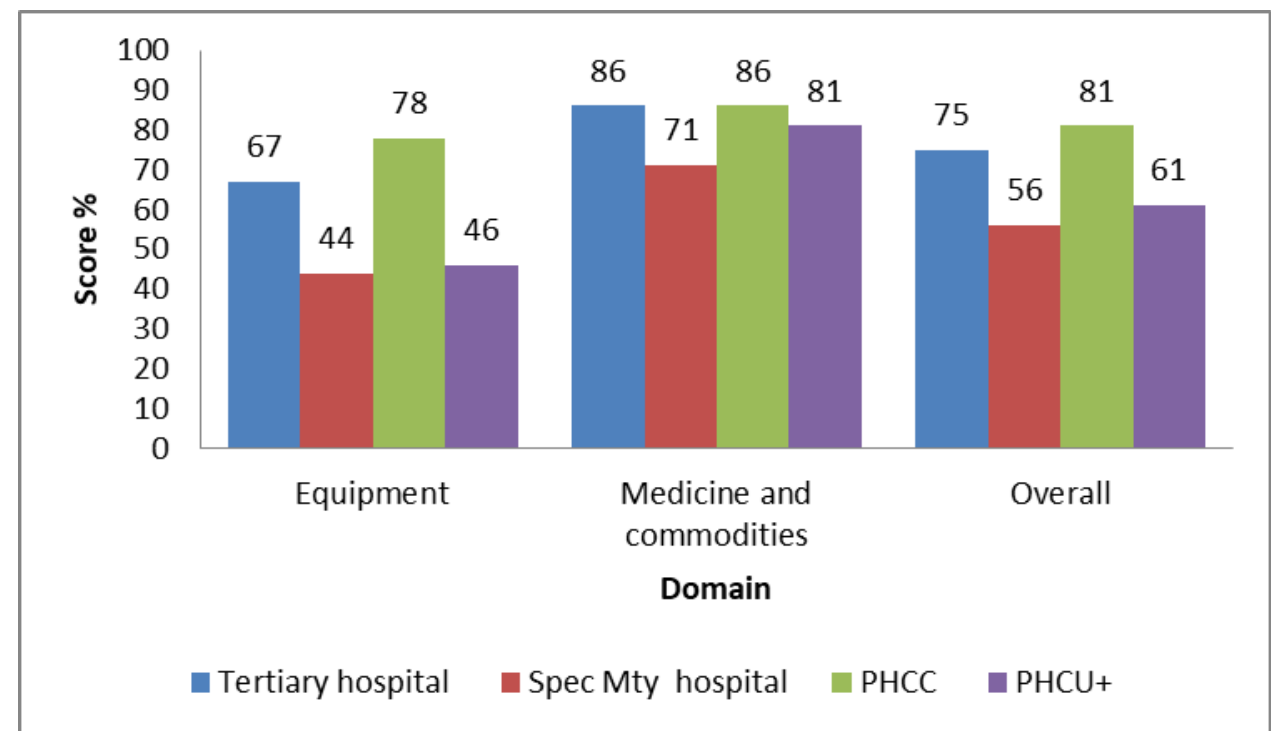

Figure 4. Readiness score for provision of delivery services according to level of health facility

\subsection{Availability of Equipment and Medicines/Commodities for Basic Delivery Services}

The overall availability was highest $(100 \%)$ for partograph, gloves, injectable uterotonic, injectable antibiotics, magnesium sulphate, and intravenous solution followed by skin disinfectants (89\%). However, some medicines and commodities like gloves, injectable antibiotics, skin disinfectants and intravenous solution were commonly available across all facilities but they were not adequate. The availability was low (22\%) for manual vacuum extractor, vacuum aspirator, dilatation and curettage (D\&C) kit. Almost all PHCU+ facilities had severe unavailability of emergency transport. Suction apparatus was available but not functioning in almost all high level facilities, while only in some few PHCU+ $3(50 \%)$, suction apparatus were available and functioning. Delivery packs and antibiotic eye ointment for newborn were not available across all surveyed facilities (Table 2).

Special maternity hospital had only 9 out of 16 key inputs for basic delivery services. There was severe unavailability of necessary delivery equipment, medicines and commodities including examination light, delivery packs, suction apparatus (mucus extractor), manual vacuum extractor, vacuum aspirator or D\&C kit, antibiotic eye ointment for newborns and diazepam injectable (Table 2). 
Table 2. Shows availability of the delivery equipment and medicines according to facility

\begin{tabular}{llllll}
\hline Delivery equipment and medicines & $\begin{array}{l}\text { All facilities } \\
(\mathbf{n}=\mathbf{9})\end{array}$ & $\begin{array}{l}\text { Tertiary hospital } \\
(\mathbf{n}=\mathbf{1})\end{array}$ & $\begin{array}{l}\text { Spec Mtyhospital } \\
(\mathbf{n = 1})\end{array}$ & $\begin{array}{l}\text { PHCC } \\
(\mathbf{n = 1})\end{array}$ & $\begin{array}{l}\text { PHCU+ } \\
(\mathbf{n}=\mathbf{6})\end{array}$ \\
\hline Emergency transport & 44 & 100 & 100 & 100 & 17 \\
Examination light & 67 & 100 & $00^{\mp}$ & 100 & 67 \\
Delivery pack & 00 & 00 & 00 & 00 & 00 \\
Suction apparatus & 33 & $00^{\text {中 }}$ & $00^{\text {中 }}$ & $00^{\mp}$ & 50 \\
Manual vacuum extractor & 22 & 100 & 00 & 100 & 00 \\
Vacuum aspirator and D\&C kit & 22 & 00 & 00 & 100 & 17 \\
Neonatal bag and mask & 78 & 100 & 100 & 100 & 67 \\
Partograph & 100 & 100 & 100 & 100 & 100 \\
Gloves & $100^{*}$ & 100 & 100 & 100 & 100 \\
Antibiotic eye ointment for newborn & 00 & 00 & 00 & 00 & 00 \\
Injectable uterotonic & 100 & 100 & 100 & 100 & 100 \\
Injectable antibiotic & $100^{*}$ & 100 & 100 & 100 & 100 \\
Magnesium sulphate & 100 & 100 & 100 & 100 & 100 \\
Diazepam injectable & 78 & 100 & 00 & 100 & 83 \\
Skin disinfectant & $89^{*}$ & 100 & 100 & 100 & 83 \\
IV solution with infusion kit & $100^{*}$ & 100 & 100 & 100 & 100 \\
\hline
\end{tabular}

*Available but inadequate, ${ }^{ }$Available but not functioning.

\subsection{Basic Emergency Obstetric and Newborn Care (BEmONC) Service Availability and Readiness}

Availability of basic emergency obstetric and newborn care includes seven signal functions, which are parenteral administration of antibiotics, parenteral administration of oxytocin, parenteral administration of anticonvulsants, assisted vaginal delivery, manual removal of placenta, manual removal of retained products, and neonatal resuscitation (Table 3).

Among all the surveyed facilities, $9(50 \%)$ facilities were conducting delivery and also providing EmONC services. Among them, $6(67 \%)$ were BEmONC level facilities. Of all the health facilities providing BEmONC services, none of the BEmONC facilities could offer fully functioning basic facility. The overall performance of assisted vaginal delivery was $2(22 \%)$. Some signal functions like parenteral administration of antibiotics and oxytocin drugs were available universally across all facility levels. Only $2(33 \%)$ out of six BEmONC facilities were performing removal of retained products, while other signal functions which required special skills like manual removal of placenta and assisted vaginal delivery were not performed in any of BEmONC facilities as well as in the special maternity hospital. The main reasons for non-performance of the signal functions were lack of necessary equipments, skilled personnel and cases (Table 3 ). 
Table 3. EmONC signal functions performed in health facilities $(\mathrm{N}=9)$

\begin{tabular}{|c|c|c|c|c|c|c|c|c|c|c|c|}
\hline 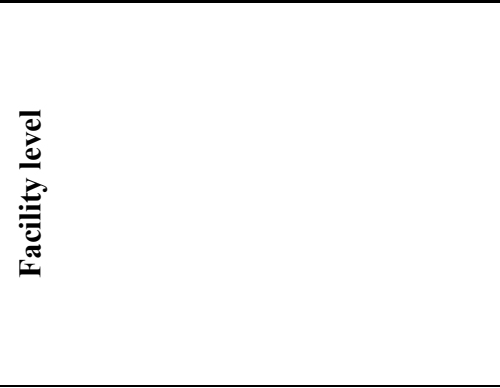 & : & 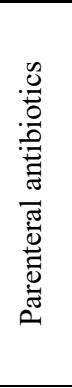 & 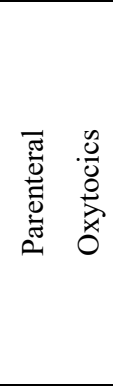 & 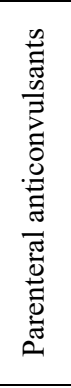 & 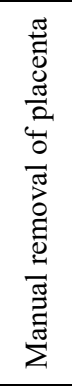 & 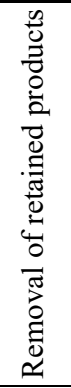 & 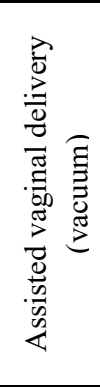 & 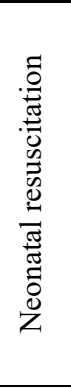 & 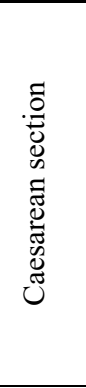 & 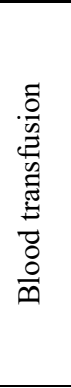 & 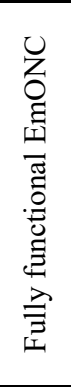 \\
\hline All & 9 & 100 & 100 & 78 & 33 & 44 & 22 & 100 & 33 & 67 & 11 \\
\hline Tertiary hospital (CEmONC) & 1 & 100 & 100 & 100 & 100 & 100 & 100 & 100 & 100 & 100 & 100 \\
\hline Special maternity hospital (CEmONC) & 1 & 100 & 100 & 100 & 100 & 00 & 00 & 100 & 00 & 00 & 00 \\
\hline PHCC(CEmONC) & 1 & 100 & 100 & 100 & 100 & 100 & 100 & 100 & 00 & 100 & 00 \\
\hline $\mathrm{PHCU}+(\mathrm{BEmONC})$ & 6 & 100 & 100 & 67 & 00 & 33 & 00 & 100 & N/A & $\mathrm{N} / \mathrm{A}$ & 00 \\
\hline
\end{tabular}

N/A because are BEmONC health facilities.

\subsection{Comprehensive Emergency Obstetric and Newborn Care (CEmONC) Services Availability and Readiness}

The overall availability of facilities which were providing CEmONC services was only $3(33 \%)$ of the all surveyed facilities. The proportion of the health facilities that offered all $9 \mathrm{CEmONC}$ signal functions that were required for emergency obstetric care services was very much lower (only 3 facilities). Only the tertiary hospital was offering all CEmONC signal functions and performed caesarean section, while $2(67 \%)$ other facilities were providing partially the required CEmONC signal functions. Neither special maternity hospital nor PHCC performed caesarean section. The reasons for non-performance of caesarean section was lack of doctors and anaesthetist (Table 3). The trained staff for performing surgery and anaesthesia were available only in the tertiary hospital (Table 4).Blood supply was available but insufficient, and CEmONC guidelines were completely lacking across all the CEmONC facilities. Unfortunately, the special maternity hospital was lacking all the 9 tracer items for CEmONC services.

Table 4. Availability of tracer items for CEmONC according to facility level $(\mathrm{N}=3)$

\begin{tabular}{llll}
\hline Tracer item for & $\begin{array}{l}\text { Tertiary hospital } \\
(\mathrm{n}=1)\end{array}$ & $\begin{array}{l}\text { Spec. Mty hospital } \\
(\mathrm{n}=1)\end{array}$ & $\begin{array}{l}\text { PHCC } \\
(\mathrm{n}=1)\end{array}$ \\
\hline CEmONC & 100 & 00 & 100 \\
Grained staff CEmONC & 00 & 00 & 00 \\
Trained staff surgery & 100 & 00 & 00 \\
Trained staff anaesthesia & 100 & 00 & 00 \\
Anaesthesia equipment & 100 & 00 & 100 \\
Incubator & 100 & 00 & 100 \\
Blood typing & 100 & 00 & 100 \\
Cross match & 100 & 00 & 100 \\
Blood sufficiency & $100^{*}$ & 00 & $100^{*}$ \\
Blood safety & 100 & NA & 100 \\
\hline
\end{tabular}

*Available but insufficient, N/A Not applicable. 


\section{Discussion}

\subsection{Availability of Healthcare Personnel}

Our study findings revealed that, the availability of healthcare personnel for providing maternal and newborns health care delivery services was the major challenge across the surveyed facilities. There is insufficiency of key healthcare personnel especially doctors who were found only in the tertiary hospital, and only one in the special maternity hospital. Nurse midwives were severely insufficient across all the surveyed facilities. This shortage might be due to lack of new employment for the key healthcare personnel needed for providing maternal and newborns healthcare services, increase in number of the health facilities providing $\mathrm{MNCH}$ services and/or improving and upgrading the facilities to the next level without considering key health healthcare personnel based on the demand.

Inadequate healthcare personnels and lack of equipment leads to inability of providing quality care to mothers and their babies (Ali \& Amer, 2018). A chronic shortage of human resources for health has a significant impact on maternal health, with increased mortality rates in the country. Healthcare personnel provides the bulk of emergency obstetric and neonatal care (Bradley \& Mcauliffe, 2009). Healthcare personnel shortage has also been reported by previous studies as a problem (Anwar et al., 2009; Banik, 2015; Kambala et al., 2011) leading to increased workloads, which in turn compromises the quality of care (Campbell et al., 2016; Graner et al., 2010). In some public sectors, the recruitment procedure for healthcare personnel is more bureaucratic and time consuming, but sometimes the recruitment procedure is obstructed for political reasons (Graner et al., 2010). Similarly, lack of human resource for health might be due to the lack of political willingness on recruiting new post for the health care personnels.

\subsection{Antenatal Care (ANC) Service Availability and Readiness}

The key findings of the study showed that, the capacity of healthcare facilities in providing maternal and newborn healthcare service in Unguja Island Zanzibar is inadequate. However, the overall availability of facilities offering ANC services for all surveyed facilities was high. The high availability of ANC services might be due to the implementation of health services delivery planning that, healthcare facilities should be available near the communities and accessed by population within five kilometres.

Overall readiness score to provide ANC services was not satisfactory. The unsatisfactory score was observed in staff training and guidelines, including medicines and commodities. The lowest service readiness scores were observed more in PHCU+ and PHCU. This might be due to poor distribution of resouces and priority in maintaining the quality and readiness of the provision of the services. Provision of healthcare services is crucial, it requires both physical presence of the services but also the quality and readiness of the services offered. This finding is consistent with other studies which showed that, readiness of ANC services was of average level, while trained staff, guidelines, equipment and supplies, diagnostics and medicines and commodities scored below average (Andriantsimietry et al., 2016; Olsen et al., 2005; Wilunda et al., 2015). Inadequate equipment and supplies have been reported to cause delays in patients to receive services, increased workload for staff, the need to source items from other facilities and causing unnecessary referral (Care et al., 2012; Mueenuddin \& Msuya, 2012; Penfold et al., 2013). The similarities of the findings might be due to their priority to cover the availability and distribution of the ANC services first to the nearby community at large. On the contrary, a study done in Kenya found that, overall readiness score for ANC was $58 \%$, ANC readiness at hospitals and ANC clinics was somewhat higher than in dispensaries, medicines/commodities and equipment domains scored highest, whereas the staff and the diagnostics domains scored lowest (MOHSW, 2013). The differences of these findings might be due to differences in healthcare budget allocations and priorities in healthcare services implementations.

Many of the health facilities rendered the ANC services in environments characterized by shortage of staff, medicines/commodities including guidelines for providing optimal quality services. Clinics thus face severe challenges in the provision of the basic RCH service packages. Furthermore, the study findings showed that, there is poor allocation of resources for the provision of maternal healthcare services. Lower level healthcare facilities (i.e PHCU and PHCU+) were provided with limited resources such as medicines and commodities including trained staff and guidelines compared to high level health facilities. Similar findings have been reported in a study conducted in peripheral Tanzania whereby it was found that there is a major challenge in the efficient delivery of commodities, inadequate staffing, a high degree of absenteeism and inadequate supervision of health facilities (Manzi et al., 2012).

\subsection{Basic Delivery Services Availability and Readiness}

The study findings revealed that, availability of basic delivery services in the surveyed facilities was not adequate. 
Only $50 \%$ of the surveyed facility offered delivery services and were more located in rural setting than in urban settings. This might have done in the move to encourage the rural community to attend for delivery services at health facilities which might reduce the home deliveries. But on the negative side, the urban community might suffer with congestions at the facilities when attending for delivery services due to scarcity of space in the facilities for delivery services. Congestions at the health facilities, might influence home delivery for the urban community.

The overall trend of health facility deliveries in Zanzibar is not satisfactory. There was decrease in facility delivery in Zanzibar from (68\%) in 2014 to (53\%) in 2015. However, there was slightly increase to (54\%) in 2016. In Unguja Island, the proportion of facility deliveries was (69\%) in 2014 to (51\%) in 2016 (HMIS, 2016). Poor distribution of delivery service availability and readiness might contributes to decrease the rate of facility delivery in Zanzibar. The Government of Zanzibar, through Ministry of health enhanced some primary health care units (PHCUs) to provide delivery services but data reveal that there was downward trend in the utilization of services in PHCUs (HMIS, 2016). Increasing facility deliveries was critical for reducing maternal mortality in Haiti (Wang et al., 2017). These findings indicate the importance of improving the availability of health facilities with provision of delivery services in Unguja urban setting.

The study findings also showed that, the overall readiness score for provision of delivery services was not satisfactory, with low readiness score in equipment, medicines and commodities. Low service readiness score for equipment was demonstrated in both special maternity hospital and $\mathrm{PHCU}+(44 \%$, and $46 \%$ respectively) while in the PHCC, the readiness score for equipment was high compared to other facilities. Low service readiness score for medicines and commodities was shown a bit lower in Special maternity hospital. This finding was consistent with findings from previous studies done in Zanzibar (Sigalla et al., 2018; Fakih et al., 2016). This might be there has been no much significant change since 2012 of the health facilities survey conducted by Fakih et al. Similarly, a study conducted in Haiti revealed that, overall, health facilities are poorly equipped and do not appear ready to provide high-quality delivery services (Wang et al., 2017). Improving the quality of care at health facilities could contribute to increased proportion of facility deliveries particularly in urban areas, where substantial number of women still deliver at home (Wang et al., 2017). It is highlighted that, inadequate availability of drugs and supplies and poor health infrastructure are responsible for the high mortality rates in some low and middle-income countries (LMIC) (Campbell \& Graham, 2006; O’Neill et al., 2013; Shadrack, 2016; Spangler, 2012; Leslie et al., 2017). This could be due to poor resources allocation within health care system or health facilities managing authorities, however both were Government health facilities.

The Ministry of Health of Zanzibar insists and encourages the women to attend in primary level facilities for delivery but, unfortunately, these facilities provide substandard care due to unavailability of enough equipment and required medicines and commodities for quality care to the mothers and newborns. Similar findings reported that, shortage of supplies, drugs and basic equipment could compromise the quality of care, motivation of staff, as well as the utilisation of services in health facilities. Weak health referral systems to support case management of complications of pregnancy inevitably reduces the overall impact of ANC (Lincetto et al., 2013).

This study finding might be significantly fuelling the poor quality maternal health services in Zanzibar which could be resulting in increasing maternal mortality rate due to lack of health care facility readiness to provide interventions, which is a minimum requirement but not a guarantee for the delivery of quality ANC services (Kanyangarara et al., 2017).

\subsection{BEmONCServices Availability and Readiness}

Our study findings showed that, despite $50 \%$ of the surveyed facilities offered delivery services, the availability of BEmONC services in Unguja Island, Zanzibar is still insufficient based on the required number per available population and also uneven distribution. The urban areas of Unguja Island have limited numbers of facilities offering BEmONC services compared to the rural areas. This might be due to the efforts addressed by the Government on expansion of these services in rural areas where home deliveries and birth complications are more pronounced than in urban areas. Similar findings were reported in a study conducted by Fakih in 2012, who found that, despite Zanzibar having met the minimum standards for EmONC availability per population, the met need is still quite low (Fakih et al., 2016).

Furthermore, our study found that, the overall readiness for provision of BEmONC services was inadequate; few (21\%) health facilities offered all BEmONC signal functions despite adequate number of health facilities designated to offer BEmOC or CEmONC services. Many health facilities do not offer all seven signal functions. This study finding also concurs with findings from other health facility surveys done in Tanzania, Uganda and Madagascar (Andriantsimietry et al., 2016; Bakari et al., 2015; MOHCDGEC, 2016; Wilunda et al., 2015). 
This study also showed that, overall, there is lack of some vital signal functions which are important for the maternal and neonatal survival whereby the least performed signal functions being assisted vaginal delivery, manual removal of placenta, manual removal of retained products and neonatal resuscitation. Primary health care units had unavailability of emergency transport and manual vacuum extractors. This implies that some emergency services were offered more in the hospital than in PHCU facilities. This might be due to lack of healthcare providers who can perform life saving procedures, or it might be due to lack of equipment required for emergency in lower level health facilities. Lack of certain services in PHCU facilities might result in women attending more in hospital facilities or more referrals to higher level, which may cause congestions, delays or inadequate delivery care for complicated cases. Similar findings have been reported in other studies which found that, manual vacuum aspiration, and assisted vaginal delivery were the least performed signal functions in health facilities (Fakih et al., 2016; Mueenuddin \& Msuya, 2012; Spangler, 2012). With inadequate implementation of the two EmOC signal functions, namely, assisted vacuum delivery and manual removal of placenta (MOHSW, 2015), meeting the target of BEmOC is a challenge. Even signal functions requiring relatively little skills such as parenteral administration of antibiotics, oxytocin and anticonvulsants are still inadequate across all health facilities. Similar findings were revealed by Ameh (Ameh et al., 2012). The availability and quality of care at facility level needs to be improved in order to reduce the number of maternal and newborn deaths (Ameh et al., 2012). Most healthcare facilities were insufficiently equipped to provide basic clinical care. If countries are to strengthen health-system capacity towards achieving universal coverage, more attention needs to be given to within-country inequities (Hsia et al., 2012; Leslie et al., 2017; O’Neill et al., 2013). Access to emergency medical care at delivery is crucial for saving lives. From the above, it means that majority of women experiencing obstetric complications do not receive care they need. Some women do not receive care because they do not deliver in a health facility but others deliver in a facility that does not provide the necessary care (Wang et al., 2017).

\subsection{CEmONCService Availability and Readiness}

This study showed that, the availability of comprehensive emergency obstetric care (CEmONC) services of the surveyed facilities were satisfactory and met the required Unite Nation (UN) standard. However, our study found that, the proportion of the health facilities that offered all 9 CEmONC signal functions that are required for emergency obstetric care services was very much lower and only the tertiary hospital offered fully all CEmONC signal functions as required. Caesarean section was performed only in the tertiary hospital out of the three CEmONC facilities because of lack of doctors and anaesthetists in the special maternity hospital and PHCC. Similarly, in a study done in Madagascar a low coverage of CEmONC with only two out of nine regional referral hospitals providing the services (Andriantsimietry et al., 2016) Also the availability of blood supply was not sufficient across all CEmONC facilities which is similar to the previous study elsewhere (Andriantsimietry et al., 2016). Availability of blood is important in delivery services and is one of the reasons leading to maternal death.

\section{Conclusion and Recommendations}

The overall readiness to provide maternal and newborn healthcare services remains unsatisfactory in the health facilities in Unguja Island, Zanzibar, despite the satisfactory availability of maternal and newborn healthcare services. There is need to strengthen the antenatal care services at PHCUs through provision of equipment and necessary medicines and commodities. Both, basic and advanced delivery services need to be improved with provision of necessary equipment and supplies at all health facility levels. The Ministry of Health should strengthen the procurement and allocation chain for equipment and necessary medicines and commodities coupled with increasing the number of required human resource for health through new employments and training. There is need to improve skills of providers to ensure that at least minimum coverage of emergency obstetric careis in place in every health facility which is conducting delivery services.

\section{Study Limitations}

- The levels of facilities were different in terms of service provision. Despite all being public health facilities, they are managed by different level of authorities, this might under estimate or exaggerate the study findings. However, authors tried to describes each level by comparing the facility levels in the findings sections.

- The selection of study units was limited only to public health facilities which might influence the study findings. However, the authors tried to balance by selecting those public facilities with high attendance of clients in order to represent other facilities to reflect the real situation in public facilities.

- The findings of this study based on the reported of data on the day of data collection including previous three months of services offered by the facility, which might influence the findings. 


\section{Acknowledgements}

The authors express their gratitude to all healthcare facility staffs, doctors in-charge who participated by providing the required information for this study.

\section{Authors' Contributions}

RRB originated the study and contributed to the study design, analysis and drafted the manuscript. RNM contributed to the design of the study and writing of the manuscript. BTM participated by providing constructive comments, ideas and reviews of the manuscript. RRB, RNM and BTM critically revised the final draft manuscript. All authors read and approved the final manuscript.

\section{Competing Interests Statement}

The authors declare that there are no competing or potential conflicts of interest.

\section{References}

Ali, N., \& Amer, H. (2018). Obstetrics Staff Nurses Expected Versus Actual Role at Maternity and Child Health University Hospital. International Journal of Nursing Science, 8(2), 27-43. https://doi.org/10.5923/j.nursing.20180802.03

Alkema, L., Chou, D., Hogan, D., Zhang, S., Moller, A., Gemmill, A., ... \& Say, L. (2016). Global , regional , and national levels and trends in maternal mortality between 1990 and 2015. The Lancet, 387, 462-474. https://doi.org/10.1016/S0140-6736(15)00838-7

Ameh, C., Msuya, S., Hofman, J., Raven, J., Mathai, M., \& Broek, N. (2012). Status of Emergency Obstetric Care in Six Developing Countries Five Years before the MDG Targets for Maternal and Newborn Health. PLoS ONE, 7(12), e49938. https://doi.org/10.1371/journal.pone.0049938

Andriantsimietry, S., Rakotomanga, R., Rakotovao, J. P., Ramiandrison, E., Razakariasy, M. E., Favero, R., ...Bazant, E. (2016). Service Availability and Readiness Assessment of Maternal, Newborn and Child Health Services at Public Health Facilities in Madagascar. African Journal of Reproductive Health, 20(3), 149-158.

Anwar, I., Kalim, N., \& Koblinsky, M. (2009). Quality of Obstetric Care in Public-sector Facilities and Constraints to Implementing Emergency Obstetric Care Services : Evidence from High- and Low-performing Districts of Bangladesh. Journal of Health Population and Nutrition, 27(2), 139-155.

Bakari, R., Damian, D., Swai, P., Makuwani, A., Mahande, M., \& Msuya, S. (2015).Assessment of Availability, Utilization and Quality of Emergency Obstetric Care in 2014 at Hai District, Northern Tanzania. Journal of Gynecology and Obstetrics, 3(3), 43-48. https://doi.org/10.11648/j.jgo.20150303.11

Banik, B. K. (2015). Availability of and accessibility to maternal healthcare services in the northern Bangladesh. American Journal of Health Research, 3(2), 63-75. https://doi.org/10.11648/j.ajhr.20150302.13

Bradley, S., \& Mcauliffe, E. (2009). Mid-level providers in emergency obstetric and newborn health care: factors affecting their performance and retention within the Malawian health system. Human Resources for Health, 7(14). https://doi.org/10.1186/1478-4491-7-14

Campbell, O., Calvert, C., Testa, A., Strehlow, M., Benova, L., Keyes, E., ... \& Bailey, P. (2016). Maternal Health 3 The scale, scope, coverage, and capability of childbirth care. The Lancet, 6736(16), 1-16. https://doi.org/10.1016/S0140-6736(16)31528-8

Campbell, O., \& Graham, W. (2006). Strategies for reducing maternal mortality: getting on with what works. Lancet, 368(9543), 1284-1299. https://doi.org/10.1016/S0140-6736(06)69381-1

Conrad, P., Allegri, M., De Moses, A., Larsson, E., Neuhann, F., Muller, O., \& Sarker, M. (2012). Antenatal Care Services in Rural Uganda : Missed Opportunities for good-quality care. Qualitative Health Research Journal, 22(5), 619-29. https://doi.org/10.1177/1049732311431897

Chi, P., Bulage, P., Urdal, H., \& Sundby, J. (2015). Barriers in the Delivery of Emergency Obstetric and Neonatal Care in Post-Conflict Africa: Qualitative Case Studies of Burundi and Northern Uganda. PLoS ONE, 10(9), e0139120. https://doi.org/10.1371/journal.pone.0139120

Fakih, B., Nofly, A., Ali, A., Mkopi, A., Hassan, A., Ali, AM., ... \& Mrisho, M. (2016).The status of maternal and newborn health care services in Zanzibar. BMC Pregnancy and Childbirth, 16(134). https://doi.org/10.1186/s12884-016-0928-6 
Graner, S., Mogren, I., Duong, L. Q., Krantz, G., \& Klingberg-allvin, M. (2010). Maternal health care professionals' perspectives on the provision and use of antenatal and delivery care: A qualitative descriptive study in rural Vietnam. BMC Public Health, 10(608). https://doi.org/10.1186/1471-2458-10-608

Herklots, T., Acht, L. Van, Meguid, T., Franx, A., \& Jacod, B. (2017). Severe maternal morbidity in Zanzibar 's referral hospital: Measuring the impact of in- hospital care. PLOS ONE, 12(8), e0181470. https://doi.org/10.1371/journal.pone. 0181470

HMIS (2016).Anual Health Bulletin.

Hsia, R., Mbembati, N., MacFarlane, S., \& Kruk, M. (2012). Access to emergency and surgical care in sub-Saharan Africa: The infrastructure gap. Health Policy and Planning, 27(3), 234-244. https://doi.org/10.1093/heapol/czr023

Kambala, C., Morse, T., Masangwi, S., \& Mitunda, P. (2011). Barriers to maternal health service use in Chikhwawa, Southern Malawi. Malawi Medical Journal, 23(1), 1-5.

Kanyangarara, M., Melinda, M., \& Walker, N. (2017). Quality of antenatal care service provision in health facilities across Sub - Saharan Africa: Evidence from nationally representative health facility assessments. Journal of Global Health, 7(2). https://doi.org/10.7189/jogh.07.021101

Leslie, H., Spiegelman, D., Zhou, X., \& Kruk, M. (2017).Service readiness of health facilities in Bangladesh, Haiti, Kenya, Malawi, Namibia, Nepal, Rwanda, Senegal, Uganda and the United Republic of Tanzania. Bull World Health Organ, 95, 738-748. https://doi.org/10.2471/BLT.17.191916

Lincetto, O., Mothebesoane-anoh, S., Gomez, P., \&Munjanja, S. (2013).Antenatal Care. International Journal of Scientific \& Technology Research, 2(2), 51-62.

Manzi, F., Schellenberg, J. A., Hutton, G., Wyss, K., Mbuya, C., Shirima, K., ... Schellenberg, D. (2012). Human resources for health care delivery in Tanzania : a multifaceted problem. Human Resources for Health, 10(1), 3. https://doi.org/10.1186/1478-4491-10-3

MoHSW. (2015). The National Road Map Strategic Plan to Improve Reproductive, Maternal, Newborn, Child \& Adolescent Health in Tanzania (2016 - 2020).

MOHSW. (2013). Tanzania Service Availability and Readiness Assessment (Sara) 2012.

MOHCDGEC. (2016). The National Road Map Strategic Plan to Improve Reproductive, Maternal, Newborn, Child and Adolescent Health in Tanzania (2016 - 2020) (One Plan II), (June), 142.

MOHSW. (2010). Assessment of the availability of health services in terms of accessibility and quality of health care.

MoHZ. (2008). Health Management Information System Unit.Health information Bulletin.

MoHZ. (2009). Comprehensive MultI Year Plan - Zanzibar 2010-2014.

MoHZ. (2016). The Zanzibar Annual Health Bulletin.

Mueenuddin, L., \& Msuya, S. (2012). Assessment of Reproductive and Child health integration in Tanzania Challenges and Opportunities.

NBS. (2012). Population and Housing Census: Mortality and Health 2012. NBS Ministry of Finance Dar es Salaam and Office of Chief Government Statistician Ministry of State, President Office, State House and Good Governance, 2015.

NBS. (2013). 2012 Population and Housing Censu: Population Distribution byAdministrative Areas. Project Report. National Bureau of Statistics.

Obi, A., Abe, E., \& Okojie, O. (2013). Assessment of Essential Obstetric Care Services in Health Care Facilities in Benin City, Edo State. IOSR Journal of Dental and Medical Sciences (IOSR-JDMS), 10(6), 33-39.

O’Neill, K., Takane, M., Sheffel, A., Abou-zahr, C., \& Boerma, T. (2013). Monitoring service delivery for universal health coverage: the Service Availability and Readiness Assessment. Bull World Health Organ, 91, 923-931. https://doi.org/10.2471/BLT.12.116798

Olsen, Ø., Ndeki, S., \& Norheim, O. (2005) Human resourse for emergency obstetric care in northern Tanzania: distribution of quantity or quaility. BMC Human Reource Health, 3(5). https://doi.org/10.1186/1478-4491-3-5

Paxton, A., Maine, D., Freedman, L., Fry, D., \& Lobis, S. (2005). The evidence for emergency obstetric care. 
International Journal of Gynecology and Obstetrics, 88(2), 181-193. https://doi.org/10.1016/j.ijgo.2004.11.026

Penfold, S., Shamba, D., Hanson, C., Jaribu, J., Manzi, F., Marchant, T., ... Schellenberg, J. (2013). Staff experiences of providing maternity services in rural southern Tanzania - a focus on equipment, drug and supply issues. BMC Health Services Research, 13(1), 61.https://doi.org/10.1186/1472-6963-13-61

Sigalla, G., Bakar, R., \& Manongi, R. (2018). Experiences of Facility-Based Delivery Services among Women of Reproductive Age in Unguja Island, Zanzibar: A Qualitative Study. Journal of Family Medicine, 5(4), 1149.

Singh, S., Darroch, J., \& Ashford, L. (2014). Adding It Up: The Costs and Benefits of Investing in Sexual and Reproductive Health 2014.United Nations Population Fund. https://doi.org/978-1-934387-04-7

Shadrack O. (2016). Provision of essential health package in public hospitals: a case of Homabay County hospitals, Kenya. Pan African Medical Journal, 24(8). https://doi.org/10.11604/pamj.2016.24.8.9280

Spangler, S. A. (2012). Assessing skilled birth attendants and emergency obstetric care in rural Tanzania: The inadequacy of using global standards and indicators to measure local realities. Reproductive Health Matters, 20(39), 133-141. https://doi.org/10.1016/S0968-8080(12)39603-4

Wang, W., Winner, M., \& Burgert-Brucker, C. R. (2017). Limited Service Availability, Readiness, and Use of Facility-Based Delivery Care in Haiti: A Study Linking Health Facility Data and Population Data. Global Health, Science and Practice, 5(2), 244-260. https://doi.org/10.9745/GHSP-D-16-00311

World Health Organization [WHO]. (2014). Reducing maternal and child mortality in Zanzibar: Wired Mothers. https://doi.org/10.1186/1471-2393-14-29.

World Health Organization [WHO]. (2015). Trends in maternal mortality: 1990 to 2015: Estimates by WHO, UNICEF, UNFPA, World Bank Group and the United Nations Population Division. Geneva.

WHO, UNFPA, \& UNICEF. (2009). Monitoring emergency obstetric care: A handbook. Geneva: WHO. https://doi.org/10.3109/01443611003791730

World Health Organization [WHO]. (2017). World Health Statistics 2017: Monitoring Health for The SDGs. World Health Organization. https://doi.org/10.1017/CBO9781107415324.004

Wilunda, C., Oyerinde, K., Putoto, G., Lochoro, P., Dall'Oglio, G., Manenti, F., ... \& Quaglio, G. (2015). Availability, utilisation and quality of maternal and neonatal health care services in Karamoja region, Uganda: a health facility-based survey. Reproductive Health, 12(30). https://doi.org/10.1186/s12978-015-0018-7

\section{Copyrights}

Copyright for this article is retained by the author(s), with first publication rights granted to the journal.

This is an open-access article distributed under the terms and conditions of the Creative Commons Attribution license (http://creativecommons.org/licenses/by/4.0/). 